\title{
Ionic and Electronic Conductivity of Nanostructured, Samaria-Doped Ceria
}

\author{
E. C. C. Souza, ${ }^{a}$ W. C. Chueh, ${ }^{b}$ W. Jung, ${ }^{b}$ E. N. S. Muccillo, ${ }^{a}$ and S. M. Haile ${ }^{b, z}$ \\ ${ }^{a}$ Energy and Nuclear Research Institute, Sao Paulo, Sao Paulo 05508-000, Brazil \\ ${ }^{b}$ California Institute of Technology, Pasadena 91125, California, USA
}

\begin{abstract}
The ionic and electronic conductivities of samaria doped ceria electrolytes, $\mathrm{Ce}_{0.85} \mathrm{Sm}_{0.15} \mathrm{O}_{1.925-\delta}$, with nanometric grain size have been evaluated. Nanostructured bulk specimens were obtained using a combination of high specific-surface-area starting materials and suitable sintering profiles under conventional, pressureless conditions. Bulk specimens with relatively high density ( $\geq 92 \%$ of theoretical density) and low medium grain size (as small as $33 \mathrm{~nm}$ ) were achieved. Electrical A.C. impedance spectra were recorded over wide temperature $\left(150\right.$ to $\left.650^{\circ} \mathrm{C}\right)$ and oxygen partial pressure ranges $\left(0.21\right.$ to $\left.10^{-31} \mathrm{~atm}\right)$. Under all measurement conditions the total conductivity decreased monotonically with decreasing grain size. In both the electrolytic and mixed conducting regimes this behavior is attributed to the high number density of high resistance grain boundaries. The results suggest a possible variation in effective grain boundary width with grain size, as well as a possible variation in specific grain boundary resistance with decreasing oxygen partial pressure. No evidence appears for either enhanced reducibility or enhanced electronic conductivity upon nanostructuring.

C 2012 The Electrochemical Society. [DOI: 10.1149/2.056205jes] All rights reserved.
\end{abstract}

Manuscript submitted December 6, 2011; revised manuscript received February 6, 2012. Published February 28, 2012. This was

Paper 722 presented at the Vancouver, Canada, Meeting of the Society, April 25-30, 2010.

The transport and redox properties of ceria have been studied extensively due to the suitability of this material to a wide range of applications including fuel cells (as both electrolyte ${ }^{1,2}$ and anode component $^{2-5}$ ) and catalytic convertors. ${ }^{6}$ In recent years there have been observations of 'non-trivial' size effects in ceria in which nanostructuring results in dramatically enhanced conductivity, particularly for grain sizes of $<30 \mathrm{~nm}^{7}$ Concomitant with this change in transport properties is a dramatic increase in reducibility, manifest as a decrease in the standard enthalpy of the reduction reaction. ${ }^{8}$

Although the details of the observations differ between authors, the grain boundary behavior of undoped ceria free of intergranular impurity phases is relatively well-explained in terms of a space-charge model. ${ }^{7,9}$ In brief, due to inherent differences in the chemical bonding environment at grain boundaries and in the bulk, the grain boundary interface or core displays a charge imbalance between anionic and cationic species leading to an interfacial space-charge potential, $\Delta \phi_{0}$. This potential, in turn, causes a redistribution of mobile species in the near vicinity of the core, i.e., the space charge region. Inferred values of $\Delta \phi_{0}$ in ceria are $\sim 0.3$ to $0.7 \mathrm{~V}$, and, being positive in sign, imply that vacancies are depleted, whereas electron concentrations, resulting from the $\mathrm{Ce}^{4+} / \mathrm{Ce}^{3+}$ equilibrium, are enhanced in the space charge region. The width of the space-charge zone (the effective grain boundary thickness) typically falls between 1.2 and $6 \mathrm{~nm}$. For microcrystalline materials, the consequence is that the grain boundaries serve to block the motion of the predominately mobile oxygen vacancies as they traverse the depletion region and migrate from one grain to the next. For nanocrystalline materials, because the volume fraction of grain boundaries is large, substantial electron transport along grain boundaries, by-passing the grain interiors, can occur. In an impedance measurement, this electrondominated case reveals itself as a single arc in the Nyquist representation (where electrode behavior is excluded) ${ }^{8,10}$ Microcrystalline materials, in contrast, generally display two impedances arcs, with the higher frequency arc attributable to bulk (grain interior) transport and the lower frequency arc to transport across high impedance grain boundaries. ${ }^{11,12}$

The case of heavily doped ceria is less clear, and the experimental results somewhat contradictory, Table I. Here, only results for bulk, as opposed to thin-film, materials are considered because of the potential impact of the film-substrate interaction on transport behavior. Because the width of the space charge zone is inversely proportional to the square root of the bulk dopant concentration in the

\footnotetext{
z E-mail: smhaile@caltech.edu
}

Mott-Schottky treatment of the space model (which takes the dopant concentration to be fixed with respect to position), one expects that electron-dominated transport would emerge at relatively smaller grain sizes in doped than in undoped ceria (for the same value of $\Delta \phi_{0}$ ). In the absence of electron-dominated transport, decreases in grain size would, to a first approximation, simply increase the number density of high impedance grain boundaries and lead to a monotonic increase in overall resistance. Such behavior has been reported, for example, for microcrystalline $\mathrm{Ce}_{0.8} \mathrm{Gd}_{0.2} \mathrm{O}_{2-\delta}$ with mean grain size ranging from 3 to $17 \mu \mathrm{m} .{ }^{11}$ Beyond microstructural considerations, one can also imagine that different processing techniques would have greater influence on the grain boundary properties of doped than undoped ceria by impacting dopant profiles in the former but not the latter. Moreover, impurity effects and possible $\mathrm{H}_{2} \mathrm{O}$ incorporation at grain boundaries at the relatively low temperatures at which bulk and grain boundary transport process can both be accessed, may also impact the transport behavior.

As indicated in Table I, the early work of Chiang ${ }^{8}$ agrees with the expectation that, in contrast to undoped ceria, nanostructuring in the presence of trivalent dopants decreases overall conductivity; even for grain sizes of only $10 \mathrm{~nm}$, distinct bulk and grain boundary responses were detected in the impedance spectra with grain boundaries adding to the overall material resistance. The result is supported by more recent work from Jasinski, ${ }^{13}$ Surble et al., ${ }^{14}$ PerezColl et al., ${ }^{15}$ and Ruiz-Trejo et al. ${ }^{16}$ While the later studies have been directed toward the exploration of $\mathrm{H}_{2} \mathrm{O}$ uptake and proton transport along grain boundaries, the data nevertheless reveal that doped ceria with grain size of $\sim 100 \mathrm{~nm}$ has resistive grain boundaries. The works of Bellino et al. ${ }^{17}$ and of Anselmi-Tamburini et al. ${ }^{18}$ stand in stark contrast to these studies. For materials with sub-100 nm grains, only a single impedance arc is observed by these authors, and, in broad terms, the total conductivity increases with decreasing grain size.

The present study was carried out with the aim of shedding new light on the transport properties of nanostructured, samaria-doped ceria. A relatively mild processing approach is pursued that enables preparation of high density $\mathrm{Ce}_{0.85} \mathrm{Sm}_{0.15} \mathrm{O}_{1.925}$ (samaria-doped ceria or SDC15) with mean grain sizes of 33, 50, 61 and $73 \mathrm{~nm}$. In contrast to the majority of the previous studies, Table I, measurements are, moreover, carried out over a wide oxygen partial pressure range to reveal the possible role of electron transport and detect any enhancements in reducibility. The results are compared to those reported in two recent studies of microcrystalline SDC $15^{12,19}$ fabricated using oxide powder from the same source. 
Table I. Summary of literature reports on nanostructured GDC and SDC.

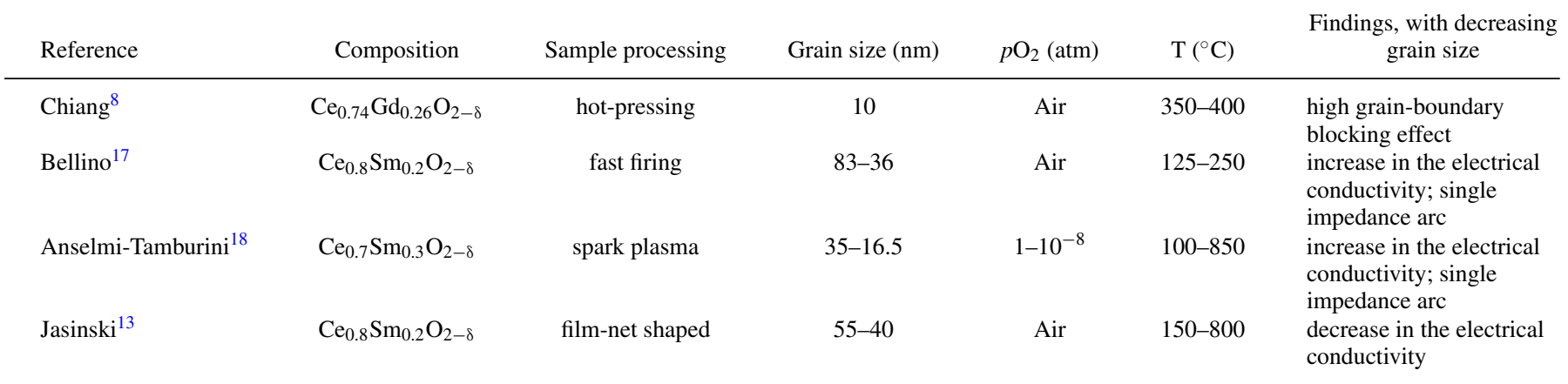

\section{Specimen Preparation and Characterization}

Nanoparticulate $\mathrm{Ce}_{0.85} \mathrm{Sm}_{0.15} \mathrm{O}_{1.925}$ (NexTech Materials Ltd) with high specific surface area $\left(168 \mathrm{~m}^{2} \mathrm{~g}^{-1}\right)$ was used as-received. This material is prepared by a hydrothermal technique, and, as reported by the manufacturer, has a silicon impurity level of $<50 \mathrm{ppm}$ and a narrow size distribution of relatively spherical primary particles of 5 to $10 \mathrm{~nm}$ in diameter. Disk-shaped specimens, $13 \mathrm{~mm}$ in diameter, were consolidated and pressed under $222 \mathrm{MPa}$ uniaxial pressure to obtain compacts $0.75 \mathrm{~mm}$ thick. In some instances a second isostatic pressing step, with a pressure in the range of 250 and $350 \mathrm{MPa}$, was also applied, increasing the green density from $\sim 3$ to $\sim 3.5 \mathrm{~g} \mathrm{~cm}^{-3}$. Sintering was carried out under ambient atmospheres, and several different heating rates, dwell temperatures, and dwell times were explored as a means of attaining high-density compacts of desired grain size. Sintered densities were determined by the water immersion method. Fractured surfaces were observed in a scanning electron microscope (LEO 1550VP Field Emission) with in-lens detectors using secondary electrons and mean effective grain size (grain boundaryto-grain boundary distance ${ }^{20}$ ) evaluated using the linear intercept method.
It was anticipated that a fast-firing procedure (fast heating, short dwell time, and fast cooling), as employed by Bellino et al., ${ }^{17}$ would be required to obtain high-density, nanostructured materials under pressureless sintering. However, after some trial and error, it was found that high densities $(>90 \%)$ and small grain sizes $(<130 \mathrm{~nm})$ could be obtained simply by using a combination of low sintering temperatures $\left(800-1000^{\circ} \mathrm{C}\right)$, moderate dwell times $(5 \mathrm{hr})$, and conventional heating rates $\left(2-5^{\circ} \mathrm{C} / \mathrm{min}\right)$. Moreover, it was found that the high ramp rates of fast-firing inevitably led, likely during the heating stage, to the formation of microcracks. Ultimately, a set of four nanostructured specimens was obtained using sintering temperatures of 800,900 , 950 and $1000^{\circ} \mathrm{C}$.

Electron micrographs of the fracture surfaces of the samples is presented in Figure 1. The materials are composed of regular polygonal grains and display relatively low porosity. Figure 2 shows the variation of the mean grain size and the relative density with the temperature of sintering. The density is relatively insensitive to temperature, falling in the range 92 to $95 \%$ of theoretical, whereas the mean grain size increases monotonically with sintering temperature, from 33 to $73 \mathrm{~nm}$ for the temperature range examined, with a distribution of grain sizes that also increases with sintering temperature. While facile
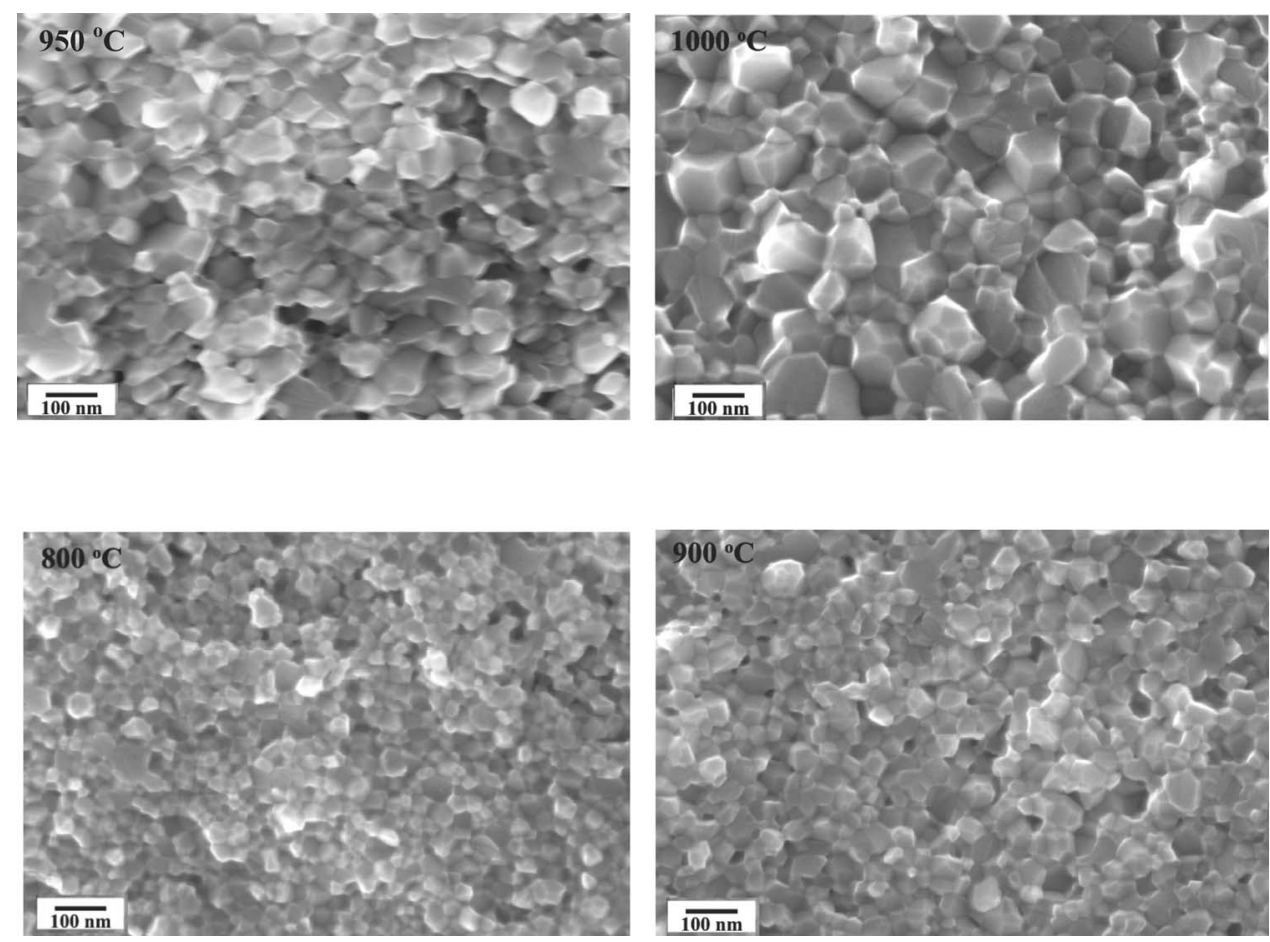

Figure 1. Scanning electron microscopy images of fracture surfaces of nanostructured SDC15 samples sintered at the temperatures indicated. 


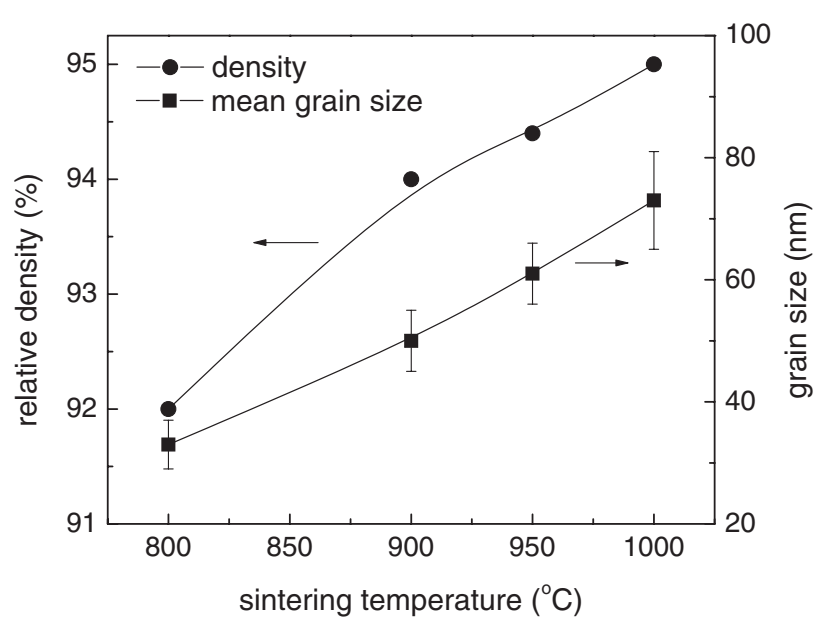

Figure 2. Relative density and mean grain size (grain boundary-to-grain boundary distance) as functions of sintering temperature in nanostructured SDC15. Error bars for grain size data reflect distribution in measured sizes. Lines are guides to the eye.

densification in the absence of significant grain growth, as reported here, is rather unexpected, it is noteworthy that Ruiz-Trejo et al. ${ }^{16}$ obtained generally similar results using starting materials from the same manufacturer. Furthermore, in contrast to spark plasma or hot isostatic pressure sintering, conventional sintering does not reduce the material and hence does not require an additional anneal in oxygen or air prior to electrical characterization. ${ }^{18}$

For the measurement of electrical transport properties, metallic electrodes were applied to the opposing sides of the compact disk samples. For the $73 \mathrm{~nm}$ and $61 \mathrm{~nm}$ grain-sized samples sintered at 1000 and $950^{\circ} \mathrm{C}$, respectively, platinum paste (6082, Engelhard), which requires an additional firing step at $900^{\circ} \mathrm{C}$, was utilized. For the smaller grained samples, sintered at 900 and $800^{\circ} \mathrm{C}$, silver paste which requires a curing step at only $300^{\circ} \mathrm{C}$, was employed instead, in order to prevent any microstructure change during specimen preparation. Gold leads supported onto a silver mesh were affixed on both faces of the pellets to serve as current collectors. Impedance data were collected using a Solartron 1260 analyzer in a frequency range from 0.01 or $0.1 \mathrm{~Hz}$ to $10 \mathrm{MHz}$ with $100 \mathrm{mV}$ of applied voltage. Equivalent circuit fitting to the impedance data was performed using the commercial software package ZView (Scribner Associates).

Measurements were carried out under a range of oxygen partial pressures and temperatures. Under synthetic air $\left(p \mathrm{O}_{2}=0.21 \mathrm{~atm}\right)$ the temperature was varied from 150 to $650^{\circ} \mathrm{C}$ in $25-50^{\circ} \mathrm{C}$ increments with a dwell time of $\sim 30$ mins at each temperature to achieve equilibrium. Under more reducing conditions ( $p \mathrm{O}_{2}=10^{-4}$ to $10^{-31} \mathrm{~atm}$ ) only high temperature data were collected due to the much longer equilibration times required. Low oxygen partial pressures were attained using $\mathrm{H}_{2} / \mathrm{H}_{2} \mathrm{O} / \mathrm{Ar}$ mixtures. At each temperature and oxygen partial pressure condition, the system was considered equilibrated when the impedance data were unchanged within $2 \%$ between sequential measurements separated in time by 5 minutes.

\section{Data Analysis Methodology}

As is shown below, the impedance spectra at low temperatures $\left(p \mathrm{O}_{2}\right.$ $=0.21 \mathrm{~atm}$ ) displayed two electrolyte-related arcs in the Nyquist representation $\left(-Z_{\mathrm{i}}(\omega)\right.$ vs. $Z_{\mathrm{r}}(\omega)$, where $Z_{\mathrm{i}}$ is the imaginary component of the impedance, $Z_{\mathrm{r}}$ is the real component, and $\omega$ is frequency), in which the high-frequency arc could be attributed to the bulk response and the low frequency arc to the grain boundary response. Accordingly, the data were analyzed in the framework of the brick layer model, ${ }^{10,11,21}$ in which the grain boundary arc is understood to result from high impedance grain boundaries in series with the lower impedance grain interiors. As the densities of all samples was comparable, Figure 2, no correction for porosity was applied.

Consistent with the brick layer model, the impedance behavior was represented using two parallel (RQ) circuits placed in series, where $\mathrm{Q}$ is a constant-phase-element (CPE) that accounts for non-ideal capacitance and a distribution of physical properties. The data were analyzed using the following quantities and relationships applicable to the brick layer model:

Impedance of a constant phase element:

$$
Z_{\mathrm{CPE}}(\omega)=Y^{-1}(j \omega)^{-n}
$$

where $\mathrm{j}=\sqrt{ }-1, Y$ and $n$ are constants, and $0 \leq n \leq 1$.

Effective capacitance of a parallel $(R Q)$ circuit:

$$
C=Y^{1 / n} R^{(1 / n-1)}
$$

valid when $\mathrm{n} \geq \sim 0.8$.

Total or effective grain boundary conductivity:

$$
\sigma_{g b}^{t o t}=\frac{1}{R_{g b}}\left(\frac{L}{A}\right)
$$

where $R_{\mathrm{gb}}$ is the absolute resistance associated with the grain boundary arc, and $L$ and $A$ are the sample length and area respectively.

Specific grain boundary conductivity:

$$
\sigma_{g b}^{s p e c}=\frac{1}{R_{g b}}\left(\frac{L}{A}\right) \frac{\delta_{g b}}{d g}=\frac{1}{\rho_{g b}^{s p e c}}
$$

where $\delta_{\mathrm{gb}}$ is the grain boundary thickness, $d_{\mathrm{g}}$ is the grain size, and $\rho_{g b}^{s p e c}$ is the specific grain boundary resistivity.

Total material resistivity:

$$
\rho^{t o t}=\rho_{g i}+\rho_{g b}^{t o t}=\rho_{g i}+\rho_{g b}^{s p e c} \frac{\delta_{g b}}{d g}
$$

where $\rho^{\text {tot }}$ is obtained from the total sample resistance and the macroscopic dimensions and the quantity $\rho_{g b}^{s p e c} \cdot \delta_{g b}$ can be defined as the impedance per grain boundary traversed (an area normalized quantity).

Dimensionless microstructural parameter:

$$
\frac{\delta_{g b}}{d_{g}}=\frac{C_{g i}}{C_{g b}}
$$

where $C_{\mathrm{gi}}$ is the absolute capacitance associated with the bulk arc, $C_{\mathrm{gb}}$ is the absolute capacitance associated with the grain boundary arc, and expression 6 is obtained under the assumption that the dielectric constants in the bulk and grain boundary regions are the same. From expressions 4 and 6 , it follows that the microstructure independent property, $\sigma_{g b}^{s p e c}$, can be obtained from an impedance measurement carried out over a frequency range that yields all the electrical parameters associated with both the bulk and grain boundary responses. Using expression 5 and an independent measurement of the grain size, it is possible to estimate the grain boundary thickness.

In addition to microstructural considerations, the electrical conductivity of trivalent-doped ceria is described by contributions from multiple carrier types. As discussed in many sources, ${ }^{1,19}$ over a relatively wide oxygen partial pressure range, the ionic contribution is independent of $\mathrm{pO}_{2}$, whereas the electronic contribution is proportional to $\mathrm{pO}_{2}^{-1 / 4}$. The $p \mathrm{O}_{2}$ dependence of the total conductivity is then described as

$$
\sigma^{t o t}=\sigma_{i o n}+\sigma_{e l}^{0}\left(p \mathrm{O}_{2}\right)^{-1 / 4}
$$

where $\sigma_{i o n}$ and $\sigma_{e l}=\sigma_{e l}^{0}\left(p \mathrm{O}_{2}\right)^{-1 / 4}$ correspond, respectively, to the ionic and electronic components of the conductivity. From a fit to Eq. 7 using data collected over a wide $p \mathrm{O}_{2}$ range it is thus possible to extract out these two contributions. 


\section{Results}

Ionic conductivity of SDC at ambient $\mathrm{pO}_{2}$. - At moderate temperatures $\left(150\right.$ to $\left.325^{\circ} \mathrm{C}\right)$ and under $p \mathrm{O}_{2}=0.21$, the impedance spectra of all four samples displayed two arcs in addition to a low frequency electrode response, as shown, for example, at $300^{\circ} \mathrm{C}$ in Figure 3. It is immediately evident, and shown explicitly in Figure 4, that the magnitude of the resistance associated with the high-frequency arc, reflecting the bulk response, is independent of grain size (Fig. 4a). In contrast, that associated with the low-frequency arc, reflecting the grain boundary response, increases sharply with increasing grain size (Fig. 4b). Thus, the total material conductivity strongly decreases upon nanostructuring. The result is in general agreement with the early work by Chiang ${ }^{8}$ and most recent work by Jasinski, ${ }^{13}$ and contradicts the results of Bellino ${ }^{17}$ and of Anselmi-Tamburini ${ }^{18}$ who observed impedance spectra with single arcs and overall increasing conductivity with decreasing grain size, Table I.

The general impact of temperature on the characteristics of the impedance spectra are presented in Figure 5, using the sample with $73 \mathrm{~nm}$ grain size as an example. With increasing temperature, a smaller and smaller portion of the high-frequency arc is accessible, a direct consequence of the exponential increase with temperature of the characteristic frequency for bulk transport. The higher frequency arc was generally inaccessible for all samples at temperatures of $350^{\circ} \mathrm{C}$ and higher, limiting the evaluation of the impedance-derived microstructural parameters to lower temperatures. Because of these frequency limitations and the significance of stray inductance effects above $325^{\circ} \mathrm{C}$, even the grain boundary arc is difficult to evaluate and, accordingly, only the sum of the (total) grain boundary and grain interior resistances are reported for higher temperatures.

The temperature dependences of the bulk, effective grain boundary and total (high temperature) conductivities are presented in Figure 6 in Arrhenius form and selected properties obtained from the impedance analysis are summarized in Table II. In both the figures and the table, the results are compared to those of the microcrystalline material. ${ }^{12,19}$ The relative dielectric constant associated with the high frequency response, ranging from $\sim 75$ to $\sim 120$, is consistent with identification of this arc as the bulk response, ${ }^{22}$ although the reasons for the observed variations in $\varepsilon_{\mathrm{r}}$ with grain size are unknown. Consistent with the behavior at $300^{\circ} \mathrm{C}$ (Fig. 3), the bulk conductivity is independent of grain size, over the entire temperature range. Furthermore, both the absolute magnitudes and the activation energies for charge transport associated with this arc, the latter set obtained from a fit of the temperature dependent behavior to the Arrhenius relation

$$
\sigma T=\sigma_{0} \exp (-E / k T)
$$

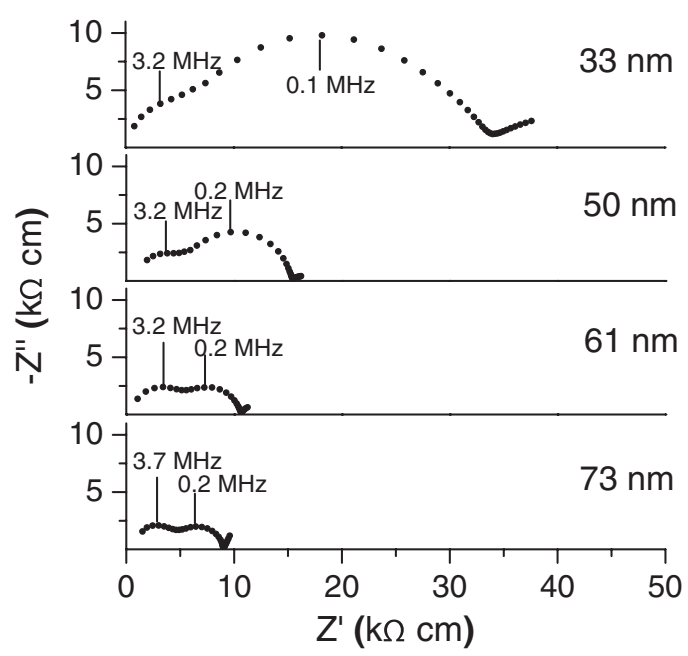

Figure 3. Representative impedance spectra collected at $300^{\circ} \mathrm{C}$ under synthetic air from nanostructured SDC15 samples with the grain sizes indicated.
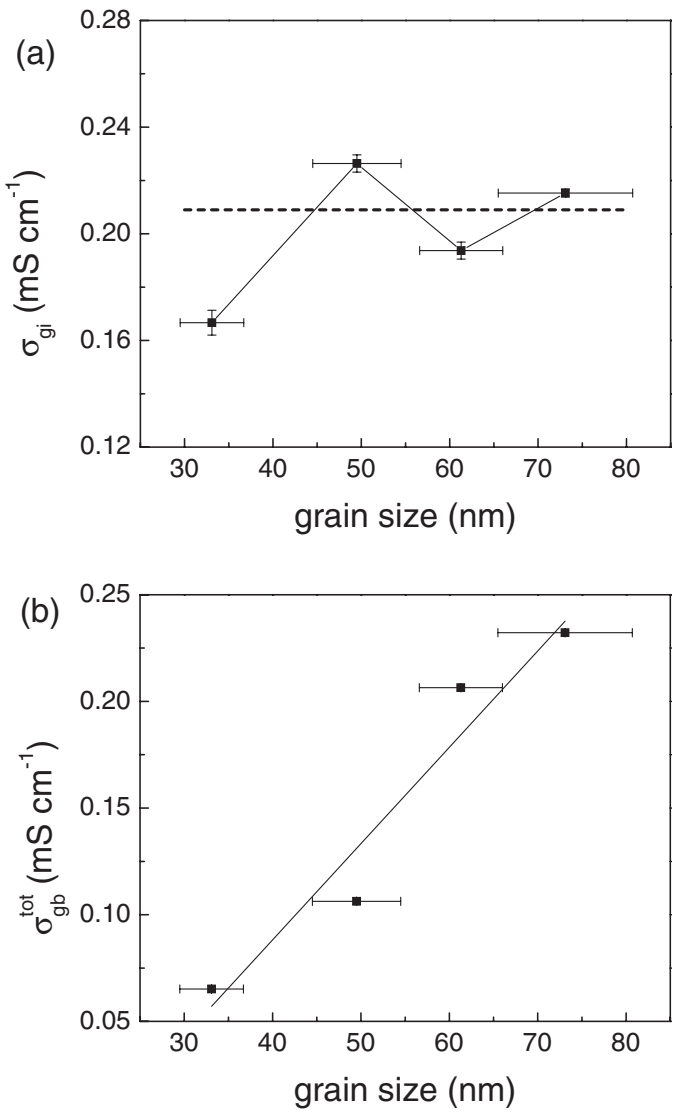

Figure 4. (a) Bulk (grain interior) and (b) effective grain boundary conductivity of nanostructured $\mathrm{SDC} 15$ at $300^{\circ} \mathrm{C}$ under synthetic air as a function of grain size. Dashed line in (a) corresponds to the microcrystalline value. ${ }^{12}$ Straight line in (b) is a linear fit to the data.
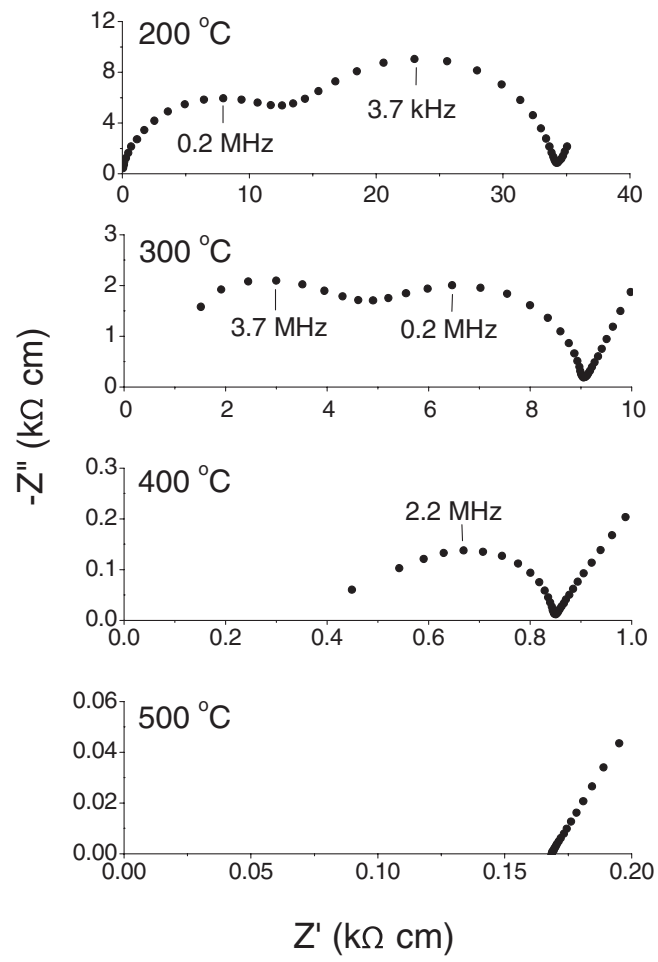

Figure 5. Representative impedance spectra collected from nanostructured SDC15 with mean grain size of $73 \mathrm{~nm}\left(1000^{\circ} \mathrm{C}\right.$ sinter $)$ under synthetic air at the temperatures indicated. 

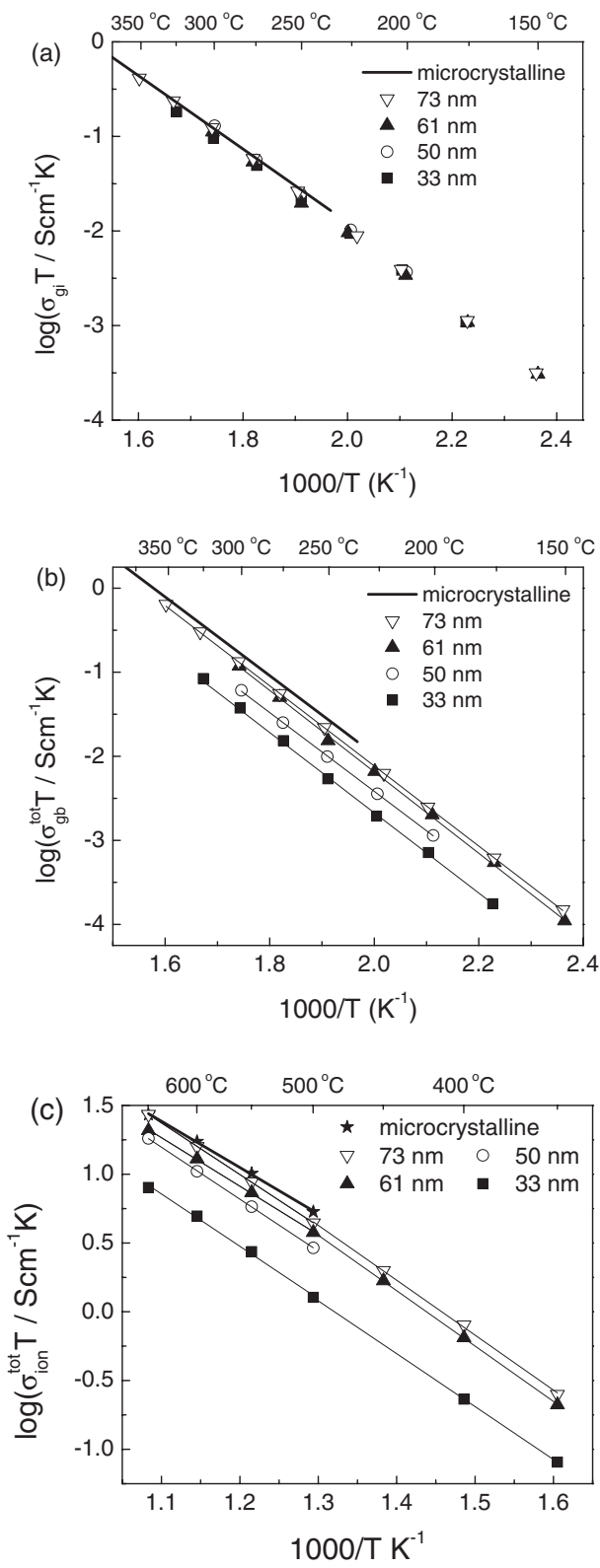

Figure 6. Temperature dependence conductivity quantities in nanostructured SDC15 under synthetic air, presented in Arrhenius form: (a) grain interior (or bulk), (b) total (or effective) grain boundary conductivity, and (c) total bulk and grain boundary conductivity. Microcrystalline data in (a) and (b) after Chueh; ${ }^{12}$ in (c) after Lai. ${ }^{19}$ are identical, within experimental error, to those of the bulk values of the microcrystalline material, ${ }^{12}$ indicating that the slight variation in dielectric behavior and slight elevation in $\varepsilon_{\mathrm{r}}$ relative to the microcrystalline value of 59 does not play a significant role in the transport properties.

The relative dielectric constant implied by the lower-frequency arc ranges from 860 to 2670 , consistent with the assignment of this response to grain boundary behavior. Moreover, the activation energy associated with this response, ranging from 0.93 to $0.95 \mathrm{eV}$ matches the microcrystalline value for grain boundary transport, further justifying the assignment to grain boundary behavior. As already noted, the mere observation of a grain boundary impedance arc implies that the boundaries act as high impedance barriers to charge transport rather than as high conductivity pathways, and, furthermore, that the grain boundary response is dominated by perpendicular rather than parallel grain boundaries. ${ }^{21}$ Consistent with this interpretation, both the effective grain boundary conductivity, Figure $6 \mathrm{~b}$, and the total conductivity, Figure $6 \mathrm{c}$, decrease monotonically with decreasing grain size. Moreover, the total conductivities observed at high temperatures are lower than those of the microcrystalline analog, ${ }^{19}$ whereas the activation energies for ion transport are comparable or slightly higher. The grain size dependence of total conductivity indicates that the grain boundary contributions to resistance are non-negligible for all four nanostructured samples, even at temperatures as high as $650^{\circ} \mathrm{C}$, despite the higher activation energies for grain boundary transport. The slight non-linearity in some of the Arrhenius plots may result from increasing dominance of the total transport behavior by grain boundaries as temperature is lowered.

Because the mean grain sizes of the samples are known from the microstructural evaluation, it is possible to directly evaluate the impedance per grain boundary within the context of the brick layer model. As implied by its definition, a plot of $\rho_{g b}^{t o t}$ vs. $1 / d_{g}$, for a series of samples in which only the grain size differs, will be linear with a slope corresponding to $\rho_{g b}^{s p e c} \cdot \delta_{g b}$ and will pass through the origin. At lower temperatures the $\rho_{g b}^{t o t}$ values are available directly from the impedance analysis. At higher temperatures, $\rho_{g b}^{t o t}$ is obtained from the difference between $\rho^{t o t}$, as measured here, and $\rho_{g i}$, as reported for microcrystalline SDC15. ${ }^{19}$ In the microcrystalline case, the data are presumed to be dominated by bulk transport behavior as a result of the large grain size and the relatively high temperature of measurement. The results, Figure 7, suggest that, in fact, the impedance per grain boundary increases with decreasing grain size. At $300^{\circ} \mathrm{C}$ (Figure 7a) the increase from the $73 \mathrm{~nm}$-grained sample to the $33 \mathrm{~nm}$-grained sample can be computed directly and is found to be about $60 \%$, from $315 \mathrm{k} \Omega \mathrm{cm}^{2}$ to $510 \mathrm{k} \Omega \mathrm{cm}^{2}$. At higher temperatures, because of possible batch-to-batch variations in the vendor supplied SDC15 and increasing significance of inductance effects at high temperatures, the numerical values are not provided. Nevertheless, the trend toward higher impedance per grain boundary with decreasing grain size is clearly evident, Figure $7 \mathrm{~b}$. If one accepts the microstructural simplifications of the brick layer model, there are two possible limiting

Table II. Selected physical and transport characteristics of nanostructured (this work) and microcrystalline ${ }^{12,19}$ SDC15; dielectric constants averaged over $200-300^{\circ} \mathrm{C}$, activation energies for grains $\left(E_{\mathrm{gi}}\right)$ and grain boundaries $\left(E_{\mathrm{gb}}\right)$ from 175 to $325^{\circ} \mathrm{C}$, and for total ionic conductivity $\left(E_{\text {tot }}\right)$ and electronic conductivity $\left(E_{\text {eon }}^{0}\right)$ from 500 to $650^{\circ} \mathrm{C}$. Quoted errors for grain size and dielectric properties reflect the measured distribution, for activation energies they reflect statistical errors obtained from fitting to an Arrhenius expression.

\begin{tabular}{|c|c|c|c|c|c|c|c|c|}
\hline $\mathrm{T}_{\text {sinter }},{ }^{\circ} \mathrm{C}$ & grain size, $\mathrm{nm}$ & $\varepsilon_{r}(g i)$ & $\varepsilon_{r}^{e f f}(g b)^{\mathrm{a}} \times 10^{-2}$ & $\frac{C_{g i}}{C_{g b}} \times 10^{2}$ & $E_{\mathrm{gi}}$ & $E_{\mathrm{gb}}$ & $E_{\mathrm{tot}}$ & $E_{\text {eon }}^{0}$ \\
\hline 800 & $33 \pm 4$ & $120 \pm 10$ & $8.8 \pm 0.3$ & $14.0 \pm 1.1$ & $0.79 \pm 0.01$ & $0.95 \pm 0.01$ & $0.76 \pm 0.03$ & $1.32 \pm 0.04$ \\
\hline 900 & $50 \pm 5$ & $79 \pm 7$ & $13.8 \pm 0.2$ & $5.7 \pm 0.5$ & $0.83 \pm 0.01$ & $0.93 \pm 0.01$ & $0.75 \pm 0.01$ & $1.62 \pm 0.03$ \\
\hline 1000 & $73 \pm 8$ & $76 \pm 4$ & $26.7 \pm 0.4$ & $2.86 \pm 0.14$ & $0.82 \pm 0.01$ & $0.95 \pm 0.01$ & $0.74 \pm 0.01$ & $1.95 \pm 0.10$ \\
\hline 1350 & $360^{\mathrm{b}}$ & $59^{\mathrm{b}}$ & $180^{\mathrm{b}}$ & $0.328^{\mathrm{b}}$ & $0.77 \pm 0.01^{b}$ & $0.93 \pm 0.02^{b}$ & $0.67 \pm 0.01^{\mathrm{c}}$ & $2.31 \pm 0.02^{\mathrm{c}}$ \\
\hline
\end{tabular}

${ }^{\mathrm{a}} \varepsilon_{r}^{e f f}(g b)=\frac{C_{g b}}{\varepsilon_{o}} \frac{L}{A}$.

${ }^{\mathrm{b}}$ microcrystalline sample data at $250^{\circ} \mathrm{C}$, activation energies over the temperature range $250-350^{\circ} \mathrm{C}$; after Chueh. ${ }^{12}$

c after Lai. ${ }^{19}$ 

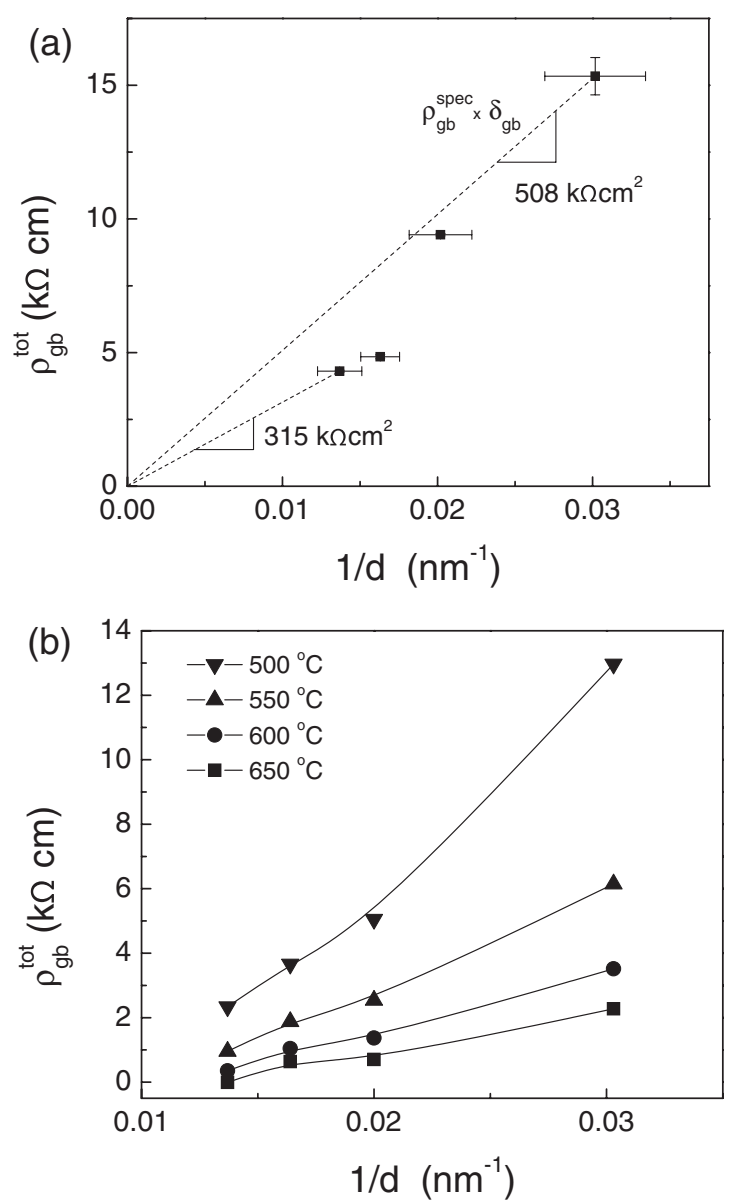

Figure 7. Variation of total (or effective) grain boundary conductivity with grain size in nanostructured SDC15 as measured under synthetic air: (a) at $300^{\circ} \mathrm{C}$ at which the grain boundary response was directly observed; and (b) at high temperatures at which the grain boundary response is estimated from the difference between the total resistance and the grain interior resistance as determined from the microcrystalline measurements. ${ }^{19}$

interpretations of this behavior: as grain size decreases the specific resistivity of the grain boundaries increases, or the grain boundary width increases.

It is possible to carry out an analogous, but independent, analysis of the dielectric behavior. Similar to the situation above, the capacitance ratio, $C_{g i} / C_{g b}$, which corresponds directly to the dimensionless microstructural parameter, $\delta_{g b} / d_{g}$, is expected to increase with decreasing grain size such that a plot of this ratio vs. $1 / d_{g}$ passes through the origin and has a slope, in this case, directly equal to the grain boundary thickness, Eq. 6 . From the summary in Table II it is apparent that $C_{g i} / C_{g b}$ increases monotonically with decreasing grain size, in general agreement with the brick layer model and grain size independent properties. However, Figure 8a, the variation with inverse grain size is once again non-linear, with behavior that mirrors that of the total grain boundary resistance. Furthermore, while the data in Figure 8 a reflect measurements at $300^{\circ} \mathrm{C}$, the behavior is replicated over the entire measurement range, Figure $8 \mathrm{~b}$. Within the framework of the brick layer model, there are again two possible limiting interpretations of this behavior: as grain size decreases the material properties change, in this case, the ratio of dielectric constants in the bulk and grain boundaries $\left(\varepsilon_{r}(g i) / \varepsilon_{r}(g b)\right.$ increases (irrespective of the absolute bulk value), or the grain boundary width simply increases. In combination with the results presented in Figure 7, it is reasonable to interpret the result in terms of behavior that is dominated by a change in grain boundary thickness, although changes in specific grain boundary properties (conductivity, dielectric constant) with changes in grain
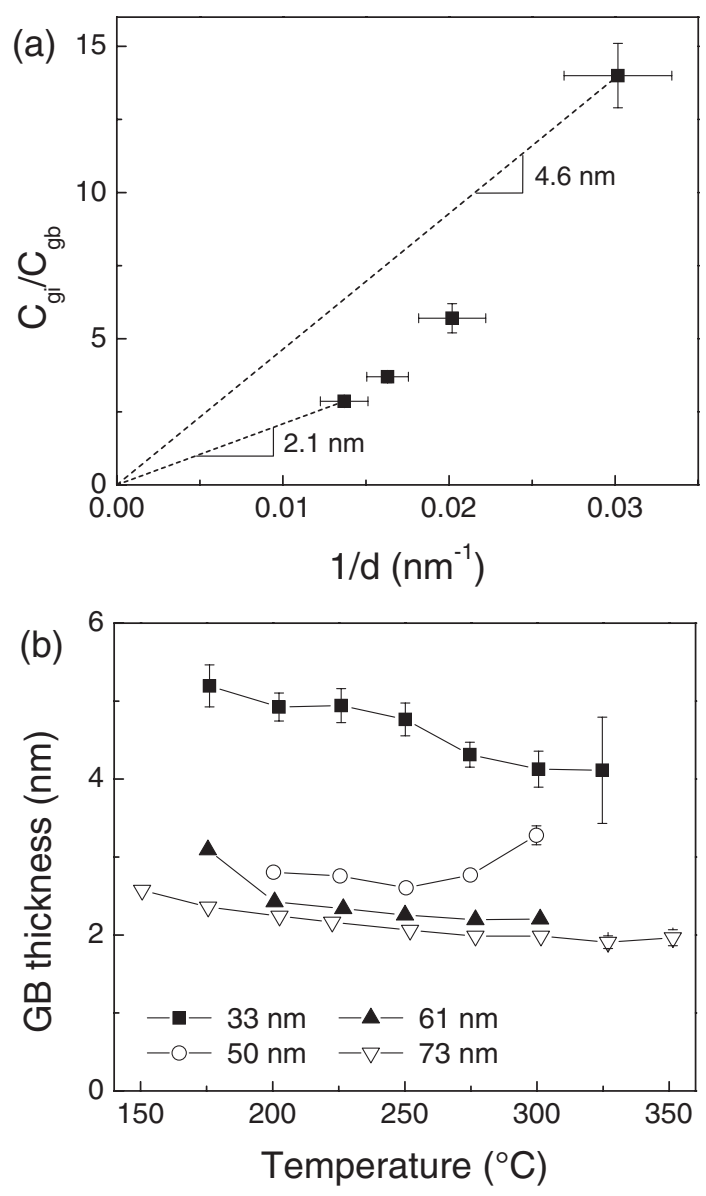

Figure 8. Evaluation of the electric behavior of nanostructured SDC15 under synthetic air: (a) the dielectric ratio as a function of inverse grain size at $300^{\circ} \mathrm{C}$; and (b) implied grain boundary thickness as a function of temperature under the assumption of spatially uniform dielectric constants.

size certainly cannot be ruled out. Under an assumption of a spatially homogeneous dielectric constant, the grain boundary thickness implied at $300^{\circ} \mathrm{C}$ decreases smoothly by a factor of $\sim 2$ from $4.6 \mathrm{~nm}$ for the smallest grained sample to $2.1 \mathrm{~nm}$ for the largest grained material. The latter value is, in turn, another factor of $\sim 2$ larger than the grain boundary thickness of the microcrystalline material, $\sim 1.2 \mathrm{~nm}^{.12}$

With the above interpretation, $C_{g i} / C_{g b}$ is taken to yield a meaningful microstructural parameter from which the specific grain boundary conductivities of the four samples can be computed according to $\sigma_{g b}^{s p e c}=\sigma_{g b}^{t o t}\left(C_{g i} / C_{g b}\right)$. For the data obtained at $300^{\circ} \mathrm{C}$, such normalization yields a specific grain boundary conductivity that is, at most, weakly dependent on grain size, Figure 9a. To minimize propagation of errors, single values of $C_{g i} / C_{g b}$ (those reported in Table II) were used for conversion over the entire temperature range. The so-normalized conductivities, Figure 9b, coincide almost precisely with one another. Moreover, the specific grain boundary conductivity is almost two orders of magnitude smaller than the bulk or grain interior conductivity, consistent with the occurrence of distinct bulk and grain boundary arcs in the impedance spectra. It is further noteworthy that the microcrystalline material displays a specific grain boundary conductivity that is almost an order of magnitude lower than that of the nanostructured material, despite comparable bulk properties.

Overall, the major conclusion to be drawn is that increasing the number density of grain boundaries in SDC15 monotonically increases the total resistivity. For the nanocrystalline series of sample, the impedance per grain boundary slightly increases with decreasing grain size. Some reports suggest enhanced grain boundary 

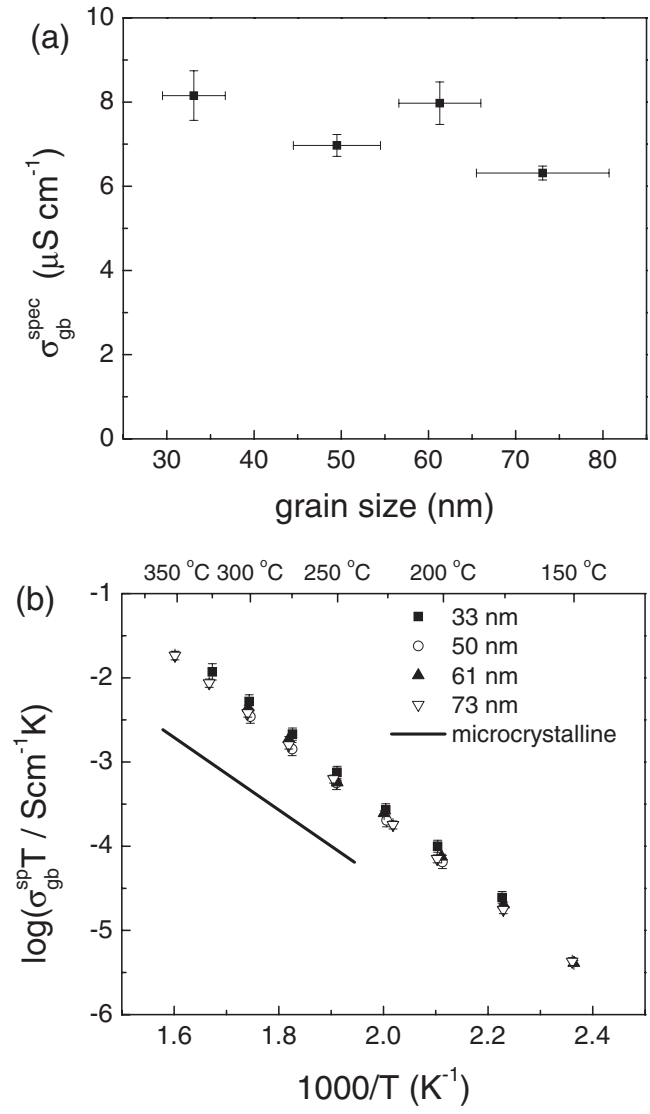

Figure 9. Specific grain boundary conductivity of nanostructured SDC15 measured under synthetic air (a) at $300^{\circ} \mathrm{C}$, and (b) as a function of temperature, presented in Arrhenius form. Microcrystalline data after Chueh. ${ }^{2}$
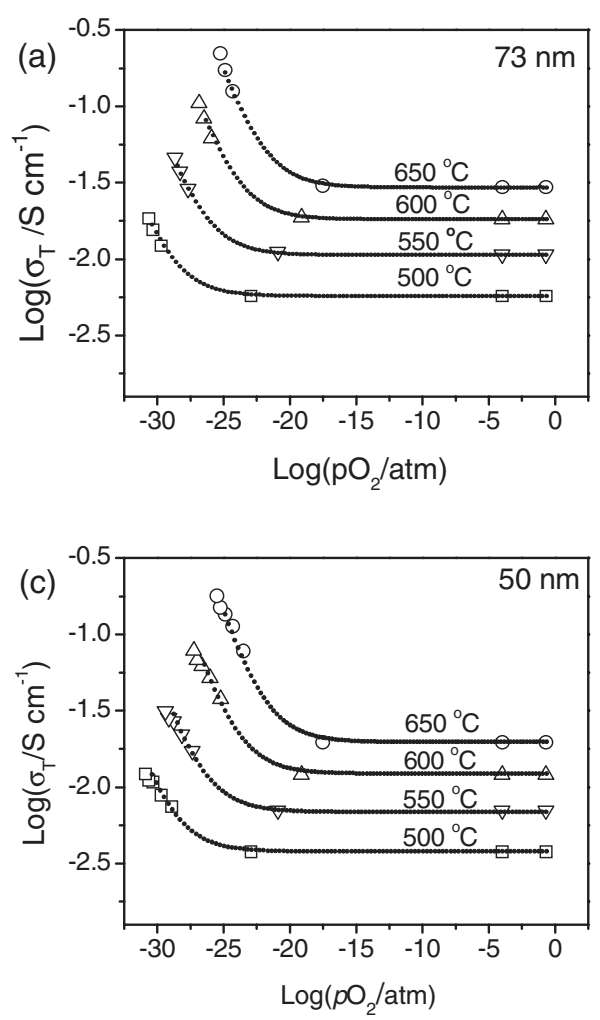

conductivity in nanostructured materials with high resistance grain boundaries due to dilution of impurities. Such behavior may explain the dramatic difference in specific grain boundary conductivity between the nanograined and microcrystalline material, but cannot be responsible for the small variation between the nanostructured samples. In sum, with the exception of an apparently increasing grain boundary thickness with decreasing grain size, as suggested independently from both the conductivity and the dielectric behavior, nanostructuring has not generated an unusual response.

Conductivity as a function of $\mathrm{pO}_{2}$.- The results of the measurements under variable oxygen partial pressure (limited to high temperatures) are summarized in Figure 10, in which the total conductivity is presented as a function of oxygen partial pressure for temperatures between 500 and $650 \mathrm{C}$ for each of the four specimens. The total conductivity in this case total implies contributions both from multiple species (ionic and electronic) and multiple processes (grain interior and grain boundary). Two features are immediately evident from the data. First, the general behavior of these materials is qualitatively unchanged from that of microcrystalline specimens, ${ }^{19}$ with a wide electrolyte regime occurring over moderate oxygen partial pressures and mixed electronic and ionic conductivity arising at low oxygen partial pressure. The latter is reflected in the increase in total conductivity with decreasing $p \mathrm{O}_{2}$. Second, on an absolute scale, the conductivity decreases with decreasing grain size, in both the electrolytic regime (as discussed above) and mixed conductivity regime. Despite this variation, the position of the electrolytic domain boundary appears unchanged.

In the mixed conducting regime the ionic conductivity can, in principle, be eliminated by evaluation of $\sigma_{\text {tot }}-\sigma_{\text {electrolytic }}$ to obtain the electronic conductivity. The analysis, Figure 11, reveals a surprising trend. Even with the electrolytic (and any other $\mathrm{pO}_{2}$ independent) contribution removed, the conductivity under reducing conditions is smallest for the smallest-grained sample. Furthermore, the exponent in the
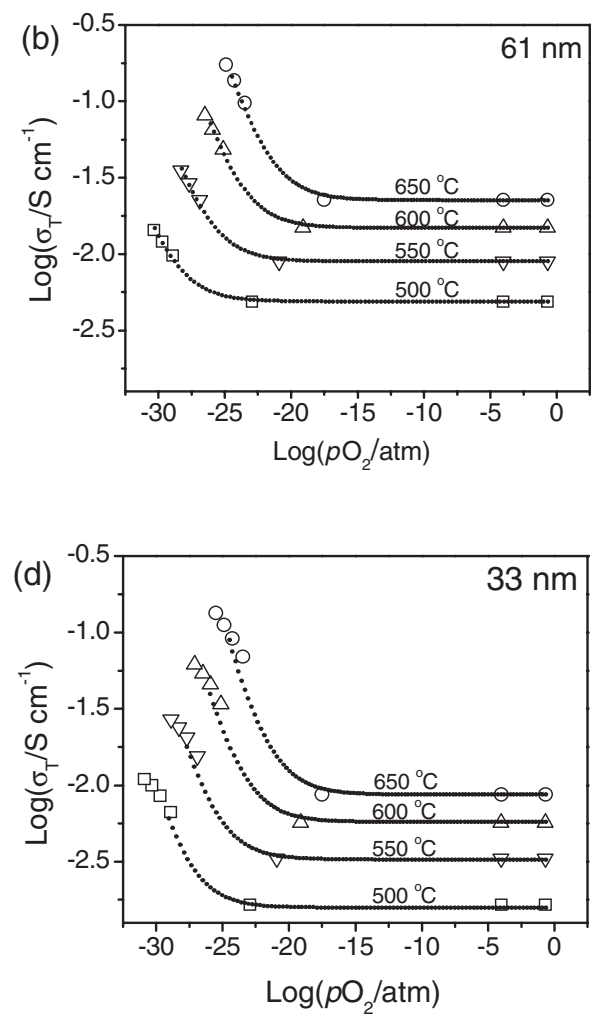

Figure 10. Total conductivity of nanostructured SDC15 as a function of temperature and of oxygen partial pressure: (a)-(d) for the four samples with grain sizes as indicated. Closely spaced points correspond to a fit to expression 7. 

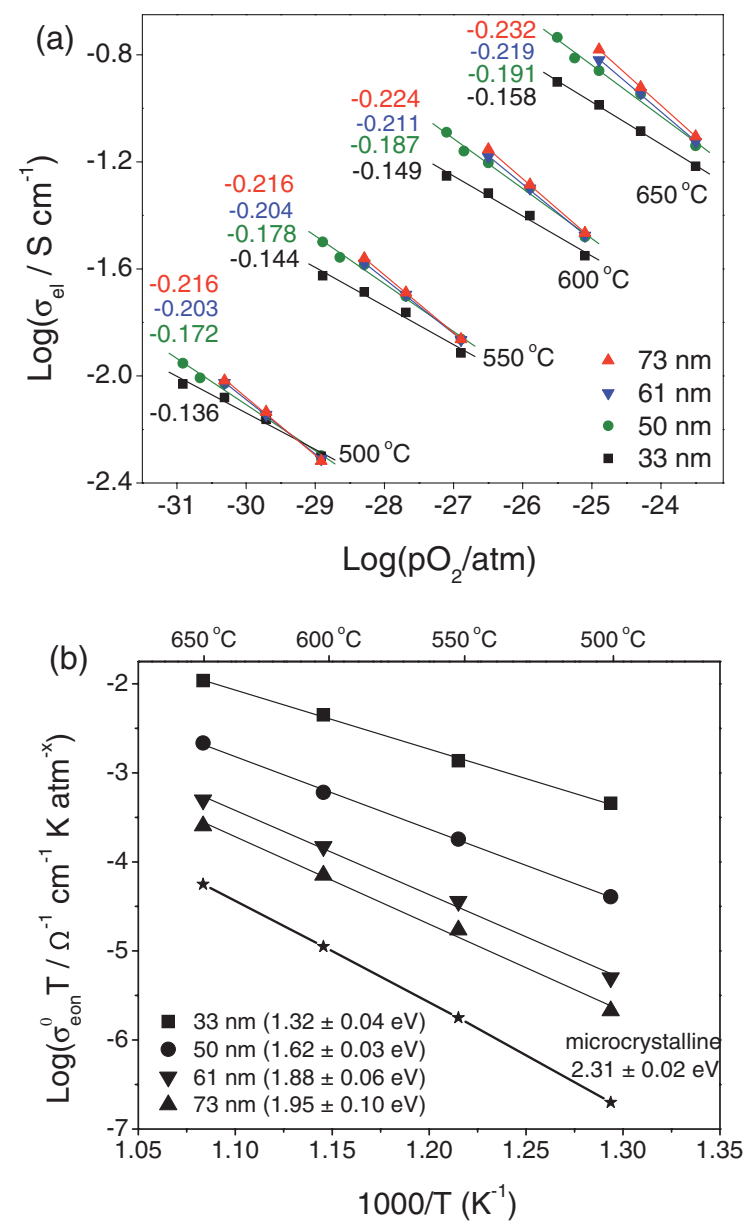

Figure 11. Electronic transport properties of nanocrystalline SDC15 in the mixed conducting regime and at high temperatures. (a) Implied electronic conductivity as a function of oxygen partial pressure (see text) and (b) implied electronic conductivity term (see text) as a function of temperature in the Arrhenius representation. In (a) quoted numbers are the slopes in the log-log representation. In (b) activation energies are as given and microcrystalline data are taken from Lai. ${ }^{19}$

power-law dependence on $p \mathrm{O}_{2} \sigma_{e l} \propto\left(p \mathrm{O}_{2}\right)^{-n}$ deviates substantially from the expected value of 0.25 , ranging from 0.136 to 0.232 , with the deviation increasing with decreasing grain size and decreasing temperature. In contrast, the microcrystalline material yields an exponent equal to 0.25 to 0.26 under similar environmental conditions. ${ }^{19}$

The temperature and oxygen partial pressure dependence of the apparent electronic conductivity is collapsed to a smaller number of curves by fitting the expression

$$
\sigma_{\text {eon }}=\sigma_{\text {eon }}^{0}(T)\left(p \mathrm{O}_{2}\right)^{-n}
$$

to the data for each temperature, where $n$ is a single, temperatureaveraged value for each specimen. The quantity $\sigma_{\text {eon }}^{0}(T)$ displays Arrhenius behavior, Figure $11 \mathrm{~b}$, and the activation energy for the apparent electronic process increases with increasing grain size, from $1.32 \mathrm{eV}$ for the smallest grained sample to 2.31 for the microcrystalline sample. ${ }^{19}$ Furthermore, the absolute value of $\sigma_{\text {eon }}^{0}(T)$ increases with decreasing grain size, but to an extent that is more than compensated for by the variation in $n$, such that smallest grained material displays the highest resistivity over the entire range of measurement conditions.

The interpretation of these trends is not obvious, but a number of possibilities, in particular within the context of the space charge model, can be considered. Several recent studies have provided compelling evidence for the applicability of the space charge model to heavily doped ceria, despite the implication of a relatively narrow space charge region $[1.2-2 \mathrm{~nm}]$. Within the context of this model, as the electron concentration increases due to low $\mathrm{pO}_{2}$, it is expected that the transport of electronic species along parallel grain boundaries would become more important, particularly for small-grained materials, potentially leading to a deviation from Eq. 7. That such a phenomenon has occurred is deemed unlikely because of the overall decrease in conductivity with decreasing grain size. An alternative possibility is that the smaller grain-sized material has entered into the electroneutrality region in which $\left[\mathrm{V}_{O}^{\bullet \bullet}\right]=2 n$, leading to $n=1 / 6$ rather than $1 / 4$, as a result of enhanced reducibility. This would be consistent with the decreasing activation energy for electronic defect creation (Figure 11b), however, it is inconsistent with the decrease in absolute conductivity with decreasing grain size and the apparent absence of a shift in the electrolytic domain boundary. Yet another candidate explanation is an increase in the barrier to ionic conductivity across grain boundaries with decreasing oxygen partial pressure. In terms of the space charge model, this is equivalent to an increase in the space charge potential, $\Delta \phi_{0}$, under increasingly reducing conditions, such that the ionic contribution to the conductivity is not fixed with $p \mathrm{O}_{2}$. Given the identical conductivities at $p \mathrm{O}_{2}=0.21$ and $10^{-4}$ atm in the present measurements (Figure 10), such an effect would apparently arise only at much lower $p \mathrm{O}_{2}$. While it is not possible to make a conclusive statement about this interpretation, it is noted that strong experimental evidence for a gas atmosphere dependence of $\Delta \phi_{\mathrm{o}}$ has been recently presented. ${ }^{12}$

\section{Conclusions}

Bulk nanostructured specimens of defect-free samaria-doped ceria were prepared by conventional sintering of cold-pressed powders of commercially available SDC15 with high specific surface area. The resulting mean grain sizes were $33,50,61$ and $73 \mathrm{~nm}$, for sintering temperatures of $800,900,950$ and $1000^{\circ} \mathrm{C}$, respectively. Under all measurement conditions (varied temperature, 150 to $650^{\circ} \mathrm{C}$, and varied oxygen partial pressure, 0.21 to $10^{-31} \mathrm{~atm}$ ), the impedance was found to increase with decreasing grain size. Under oxidizing conditions and at lower temperatures at which the bulk and grain boundary responses could be resolved, two arcs appeared in the impedance spectra. The resistance of the higher frequency, bulk (grain interior) arc was independent of grain size whereas the lower frequency, grain boundary arc increased monotonically with decreasing grain size. The impedance per grain boundary was found to increase slightly with decreasing grain size. In combination with the dielectric properties, this behavior is interpreted in terms of fixed electrical properties (conductivities and dielectric constants) and a grain boundary width that increases, implying a space charge potential that also increases, with decreasing grain size. The origin of this grain size dependent behavior is unknown, but the result points toward an increasing difference between bulk and grain boundary thermodynamics with decreasing grain size.

At high temperatures and under varied oxygen partial pressure, the nanostructured materials displayed the two regimes characteristic of microcrystalline materials, $p \mathrm{O}_{2}$-independent at higher $p \mathrm{O}_{2}$ and increasing conductivity with decreasing $p \mathrm{O}_{2}$ at lower $p \mathrm{O}_{2}$, with a boundary between these two regions also typical of microcrystalline materials. Beyond a simple reduction in the conductivity in the electrolytic $\left(\mathrm{OO}_{2}\right.$-independent) regime with decreasing grain size, even the apparent electronic conductivity decreased upon nanostructuring. This entirely unexpected and unusual behavior is interpreted not in terms of lowered electronic conductivity, but instead in terms of lowered ionic conductivity across grain boundaries. Justification for this interpretation derives from the fact that even at temperatures as high as $650^{\circ} \mathrm{C}$ the grain boundaries contribute significantly to the total resistance in the electrolytic regime. For grain boundary ionic resistance to be the cause of the unusual $\mathrm{pO}_{2}$ dependence of the total conductivity, the ionic transport must become increasingly difficult as oxygen partial pressure is lowered. In turn, this suggests that space charge effects become more severe under reducing conditions. 
In sum, we find that nanostructuring lowers the ionic conductivity of SDC15 in a monotonic fashion with decreasing grain size from 73 to $33 \mathrm{~nm}$. No evidence appears for enhanced reducibility or for enhanced electronic conductivity. The apparent decrease in electronic conductivity under reducing conditions may reflect an increasing contribution of high resistance grain boundaries to the total transport behavior.

\section{Acknowledgments}

The authors gratefully acknowledge financial support from the following agencies: the State of Sao Paulo Research Fund (FAPESP), the Brazilian National Nuclear Energy Commission (CNEN), the Brazilian National Council for Scientific and Technological Development $(\mathrm{CNPq})$, and the U.S. National Science Foundation (DMR0918224). Additional scholarship support was provided by FAPESP to E.C.C. Souza. Experimental facilities used in this work were supported the U.S National Science Foundation through the Caltech Center for the Science and Engineering of Materials, a Materials Research Science and Engineering Center (DMR-052056).

\section{References}

1. H. Inaba and H. Tagawa, Solid State Ion., 83, 1 (1996).
2. Z. P. Shao and S. M. Haile, Nature, 431, 170 (2004).

3. S. D. Park, J. M. Vohs, and R. J. Gorte, Nature, 404, 265 (2000).

4. T. Nakamura, K. Yashiro, A. Kaimai, T. Otake, K. Sato, T. Kawada, and J. Mizusaki, Journal of the Electrochemical Society, 155, B1244 (2008).

5. W. C. Chueh, Y. Hao, W. Jung, and S. M. Haile, Nature Materials, 11, 155 (2012).

6. M. S. Hegde, G. Madras, and K. C. Patil, Accounts Chem. Res., 42, 704 (2009).

7. A. Tschope, Journal of Electroceramics, 14, 5 (2005).

8. Y. M. Chiang, E. B. Lavik, I. Kosacki, H. L. Tuller, and J. Y. Ying, Journal of Electroceramics, 1, 7 (1997)

9. S. Kim and J. Maier, Journal of the Electrochemical Society, 149, J73 (2002).

10. J. H. Hwang, D. S. McLachlan, and T. O. Mason, Journal of Electroceramics, 3, 7 (1999).

11. G. M. Christie and F. P. F. vanBerkel, Solid State Ion., 83, 17 (1996).

12. W. C. Chueh, C. K. Yang, C. M. Garland, W. Lai, and S. M. Haile, Physical Chemistry Chemical Physics, 13, 6442 (2011).

13. P. Jasinski, Solid State Ion., 177, 2509 (2006).

14. S. Surble, G. Baldinozzi, M. Dolle, D. Gosset, C. Petot, and G. Petot-Ervas, Ionics, 14, 33 (2008).

15. D. Perez-Coll and G. C. Mather, Solid State Ion., 181, 20 (2010).

16. E. Ruiz-Trejo and J. A. Kilner, Journal of Applied Electrochemistry, 39, 523 (2009).

17. M. G. Bellino, D. G. Lamas, and N. E. W. de Reca, Advanced Functional Materials, 16, 107 (2006).

18. U. Anselmi-Tamburini, F. Maglia, G. Chiodelli, A. Tacca, G. Spinolo, P. Riello, S. Bucella, and Z. A. Munir, Advanced Functional Materials, 16, 2363 (2006).

19. W. Lai and S. M. Haile, Journal of the American Ceramic Society, 88, 2979 (2005).

20. Y. Yamazaki, R. Hernandez-Sanchez, and S. M. Haile, Chemistry of Materials, 21, 2755 (2009).

21. S. M. Haile, D. L. West, and J. Campbell, Journal of Materials Research, 13, 1576 (1998).

22. A. S. Nowick, A. V. Vaysleyb, and I. Kuskovshy, Physical Review B, 58, 8398 (1998) 